Proceeding Series of the Brazilian Society of Computational and Applied Mathematics

\title{
Modelo matemático simples de angiogênese tumoral com protocolos de quimioterapia
}

\section{Rafael Trevisanuto Guiraldello ${ }^{1}$}

Programa de Pós-graduação em Biometria, Unesp, Botucatu, SP

Marcelo Lobato Martins ${ }^{2}$

Departamento de Física, UFV, Viçosa, MG

Paulo Fernando de Arruda Mancera ${ }^{3}$

Departamento de Bioestatística, Unesp, Botucatu, SP

\begin{abstract}
Resumo. Apresentamos um modelo matemático baseado em equações diferenciais parciais que é aplicado na compreensão do desenvolvimento tumoral e em sua resposta a aplicação de quimioterapia. Administração do quimioterápico é em ciclos segundo dois protocolos, MTD e metronômico, bem como, em dois métodos de entrega da droga, convencional e uniforme. Através de simulações numéricas concluímos que a combinação protocolo metronômico e método de entrega uniforme é mais eficaz em reduzir a densidade do tumor durante o tratamento, mas favorece a reicidência do tumor. A combinação protocolo metronômico e método convencional acarreta em uma sobrevida maior ao paciente mesmo não sendo tão eficiente em reduzir a densidade do tumor durante o tratamento quanto a combinação anterior.
\end{abstract}

Palavras-chave. Oncologia Matemática, Câncer, Metronômico, MTD

\section{Modelo Matemático}

Denotando por $N_{1}, N_{2}, L_{1}$ e $Q$ as densidades de células neoplásicas, células normais, células endoteliais e agente quimioterápico por unidade de área, respectivamente, e baseado em [3], propomos o seguinte modelo,

\footnotetext{
${ }^{1}$ rafaeltrevisanuto@gmail.com

${ }^{2}$ mmartins@ufv.br

${ }^{3}$ pmancera@ibb.unesp.br
} 


$$
\left\{\begin{array}{l}
\frac{\partial N_{1}}{\partial t}=D_{1} \nabla^{2} N_{1}+r_{1} N_{1}\left(1-\frac{N_{1}+\alpha_{12} N_{2}}{k_{1}+L_{1}}\right)-N_{1} \mu \frac{Q}{a+Q} \\
\frac{\partial N_{2}}{\partial t}=D_{2} \nabla^{2} N_{2}+r_{2} N_{2}\left(1-\frac{N_{2}-\alpha_{21} N_{1}}{k_{2}}\right)-N_{2} \nu \frac{Q}{b+Q} \\
\frac{\partial L_{1}}{\partial t}=D_{L} \nabla^{2} L_{1}+\xi N_{1}\left(1-\frac{L_{1}}{k_{L}}\right)-\frac{\sigma}{k_{2}} L_{1}^{2}-L_{1} \eta \frac{Q}{c+Q}-\nabla \cdot\left(\chi_{\left(N_{1}, L_{1}\right)} \nabla N_{1}\right) \\
\frac{\partial Q}{\partial t}=D_{3} \nabla^{2} Q+q-\lambda Q
\end{array}\right.
$$

em que $r_{i}$ é a taxa constante de crescimento intrínseco, $k_{i}$ é a capacidade de suporte e $D_{i}$ é a constante de mobilidade aleatória da população $N_{i}(i=1,2)$. A capacidade de suporte de células neoplásicas varia com a densidade de células endoteliais $k_{1}+L_{1}$, descrevendo assim o aporte nutricional que uma neovascularização provê ao tumor. O termo de competição, dado por $\alpha_{i j}$, descreve a ação que a população $N_{j}$ exerce sobre a população $N_{i}$, em que $i \neq j(i, j=1,2)$.

O crescimento de células endoteliais é induzido pela população de células neoplásicas, com taxa intrínseca $\xi$, modelando assim a liberação de TAFs (Tumor Angiogenesis Factors [1]) na vizinhança celular e limitado pela capacidade $k_{L}$ de perfusão do tecido. A competição é dada de maneira intraespecífica, a uma taxa $\sigma$, e descreve a competição por nutrientes. $D_{L}$ é a constante de mobilidade aleatória das células endoteliais. A migração quimiotática é caracterizada pela função $\chi_{\left(N_{1}, L_{1}\right)}=\chi L_{1} /\left(k_{2}+N_{1}\right)$, que descreve o declínio da sensitividade quimiotática em elevadas concentrações de TAFs [1].

Assumimos aqui que o gradiente de concentração de células neoplásicas modela a concentração de TAFs, isto é, onde há uma maior concentração de células neoplásicas, há uma necessidade maior de nutrição, logo, há uma maior concentração de TAFs. A constante intrínseca de mobiliade quimiotática das células endoteliais é dada por $\chi$.

A infusão do agente quimioterápico, definida de acordo com [2], é dado por

$$
q=\left\{\begin{array}{ll}
q, & n<t \leq n+\tau \\
0, & n+\tau<t \leq n+T
\end{array},\right.
$$

em que $T$ é o tempo entre os ciclos de infusão do agente quimioterápico, $\tau$ é o tempo de infusão (com $T>>$ ), $q$ é uma função que caracteriza a infusão do agente quimioterápico, $\lambda$ é a constante de decaimento natural da droga e $D_{3}$ é a constante de mobilidade aleatória do agente quimioterápico. A ação do agente quimioterápico nas populações celulares é dado por uma resposta tipo Michaelis-Menten, onde $\mu, \nu$ e $\eta$ são taxas instrínsecas de ação da droga em cada população e as densidades $a, b$ e $c$ são as densidades quem ditam a ação da droga em cada população [3]. A função $q$ será dada de duas formas, apresentadas a seguir, que denominaremos de administração convencional e uniforme.

\subsection{Administração convencional}

Para a administração convencional, definimos a função $q$ como 


$$
q(x, t)=\bar{q}\left(\int_{\Omega} L_{1}(x, t) d x\right)^{-1} L_{1}(x, t)
$$

isto é, estamos administrando o agente quimioterápico com uma dose $\bar{q}$ e distribuindo esta de maneira proporcional a densidade de células endoteliais no espaço, entregando assim uma maior dose de quimioterápico onde há uma maior vascularização.

\subsection{Administração uniforme}

Para a administração uniforme, definimos a função $q$ como

$$
q(x, t)=\bar{q},
$$

ou seja, estamos distribuindo o agente quimioterápico, com dose $\bar{q}$, de uma maneira uniforme no espaço, em uma primeira tentativa em modelar a entrega do quimioterápico com a normalização da vasculatura tumoral.

\subsection{Condições iniciais e condições de contorno}

O modelo será resolvido em uma dimensão (1D), sobre o domínio $\Omega=(0, L), L=1$, com a condição inicial dada por

$$
\left\{\begin{array}{l}
N_{1}(0, x)=k_{1} e^{-20\|x-0,5\|^{2}} \\
N_{2}(0, x)=1-N_{1} \\
L_{1}(0, x)=0 \\
Q(0, x)=0
\end{array},\right.
$$

e condição de contorno, em $\partial \Omega$, dada por

$$
\frac{\partial N_{1}}{\partial n}=\frac{\partial N_{2}}{\partial n}=\frac{\partial Q}{\partial n}=\frac{\partial L_{1}}{\partial n}=0,
$$

isto é, não há fluxo das populações envolvidas e agente quimioterápico através da fronteira. A condição inicial (5) representa um tumor sólido avascular localizado no centro do tecido saudável muito próximo a sua capacidade de suporte.

\section{Simulações Numéricas}

Para o protocolo MTD utilizamos a função (2) com $T=21, \bar{q}=7200$ e $n=0, T, 2 T$ e $3 T$, ou seja, são realizadas 4 infusões do agente quimioterápico, cada infusão ocorre a cada 21 dias ( [3]).

Já para o protocolo metronômico, utilizamos a função (2) com $T=6, \bar{q}=3600 \mathrm{e}$ $n=0, T, 2 T, . ., 15 T$, ou seja, são realizadas 16 infusões do agente quimio-terápico, cada infusão ocorre a cada 6 dias ( [3]). 
(a)

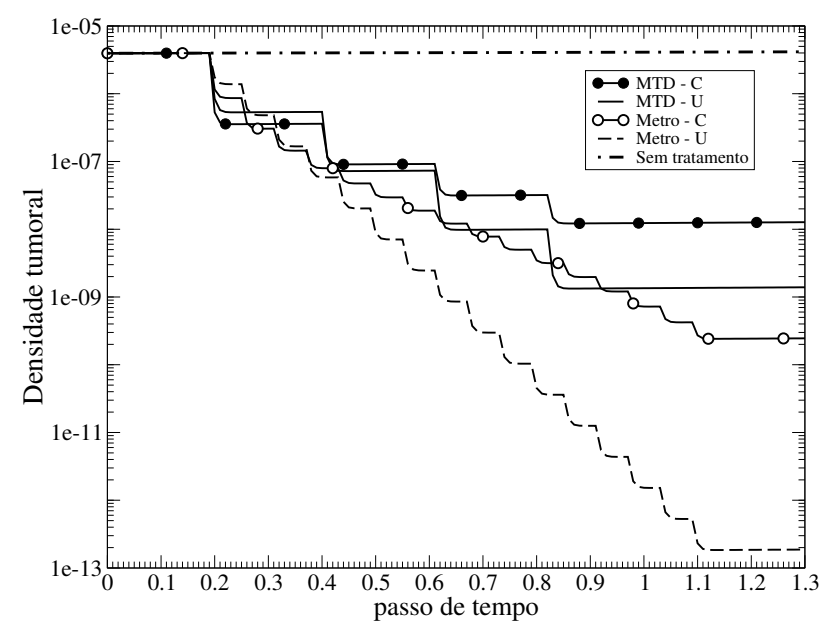

(b)

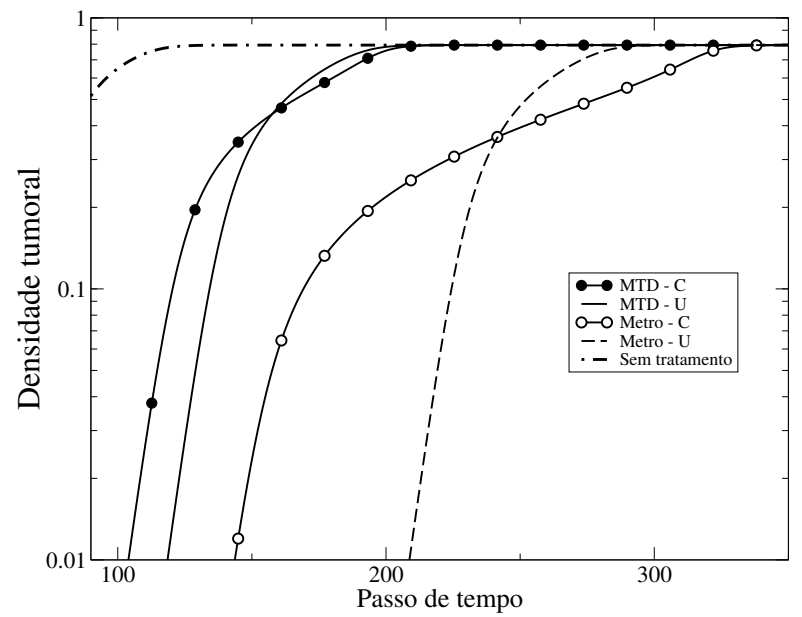

Figura 1: (a) Dinâmica do crescimento tumoral durante o tratamento e (b) até a estabilidade.

Na Figura (1)(a) ilustramos a diferença entre os protocolos de quimioterapia para cada método de entrega durante o período de tratamento junto a curva sem tratamento. Vemos que o protocolo metronômico é mais eficiente em reduzir a densidade de células tumorais durante o tratamento comparado ao tratamento MTD. Ainda, o método de entrega uniforme é mais eficaz em ambos os protocolos de quimioterapia. O método de entrega uniforme junto ao protocolo metronômico é o que mais reduz a densidade de células tumorais. Já na Figura (1)(b), ilustramos os passos de tempo até a estabilidade. O protocolo metronômico é mais eficaz, comparado ao protocolo MTD, em prolongar a estabilidade. Apesar do método uniforme junto a ambo os protocolos ser mais eficiente em reduzir a densidade de células tumorais durante o período de tratamento, este favorece a reincidência de células tumorais, pois em ambos os protocolos o método convencional se 

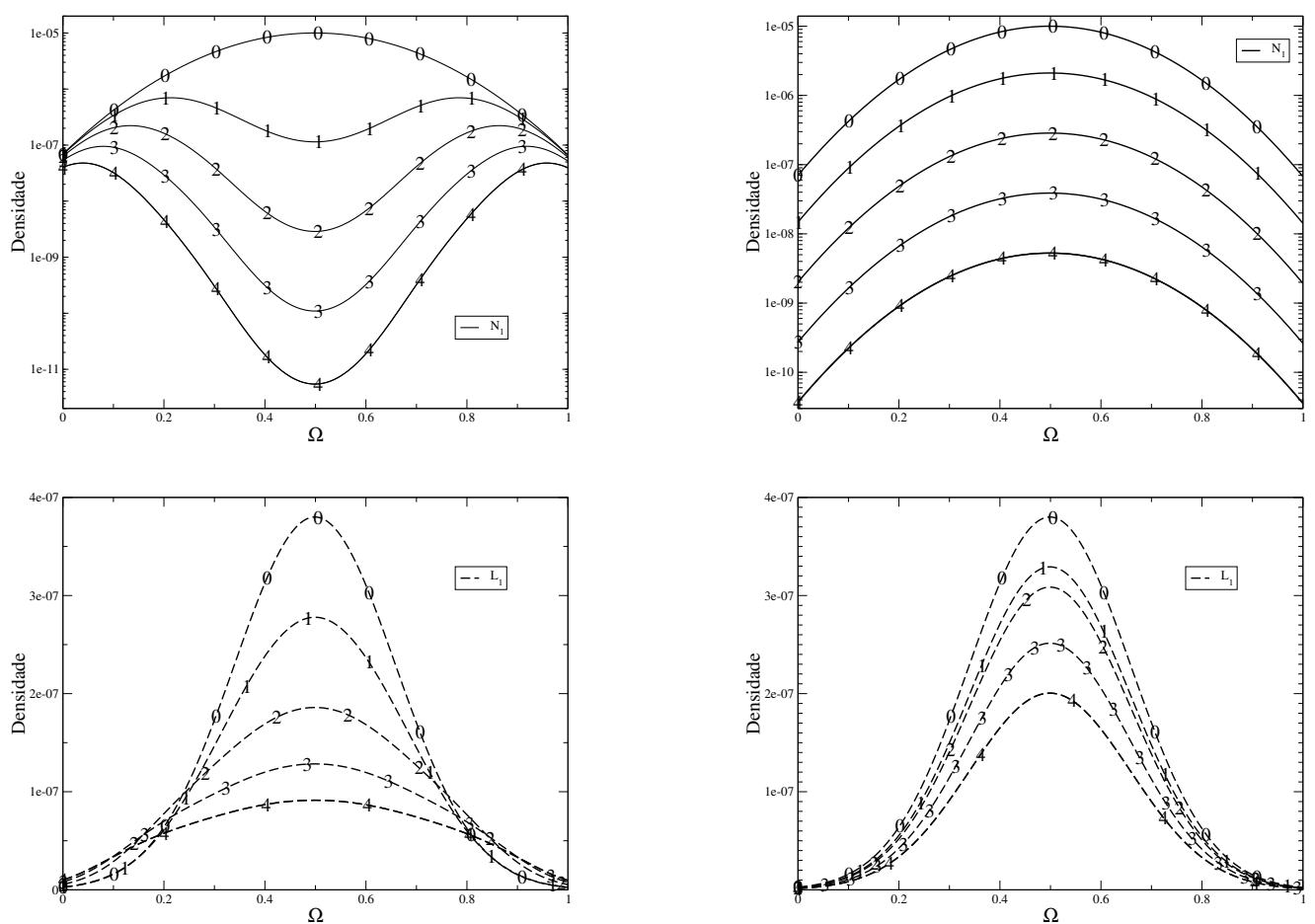

Figura 2: Densidade distribuída espacialmente durante o tratamento segundo o protocolo MTD, à esquerda para o método convencional e à direta, método uniforme.

mostrou melhor em prolongar a estabilidade.

Nas Figuras (2) e (3) exibimos espacialmente como a densidade de células tumorais e endoteliais se comportam durante o tratamento. A distribuição da densidade no espaço logo após o tratamento do método uniforme induz o crescimento mais rápido de células endoteliais, comparado com o método convencional. Assim, a densidade células endoteliais cresce, aumentando o suporte de células tumorais, favorecendo o rápido crescimento do tumor.

\section{Conclusões}

Apresentamos um modelo matemático de equações diferenciais parciais que descreve o crescimento de um tumor sólido a simulamos numéricamete a combinação entre dois protocolos de quimioterapia, MTD e metrômico, e dois métodos de entrega, convencional e uniforme. Inicialmente concluímos que o protocolo metrômico é mais eficiente em reduzir a densidade de células neoplásicas durante o período de tratamento, independente do método de entrega. A combinação protocolo metronômico e método uniforme é a mais eficente em reduzir a densidade de células neoplásicas, mas esta favorece a reincidência do tumor. Já o protocolo metronômico junto ao método convencional é o eficiente em produzir uma sobrevida, apesar de não ser o mais eficiente durante o período de tratamento. 

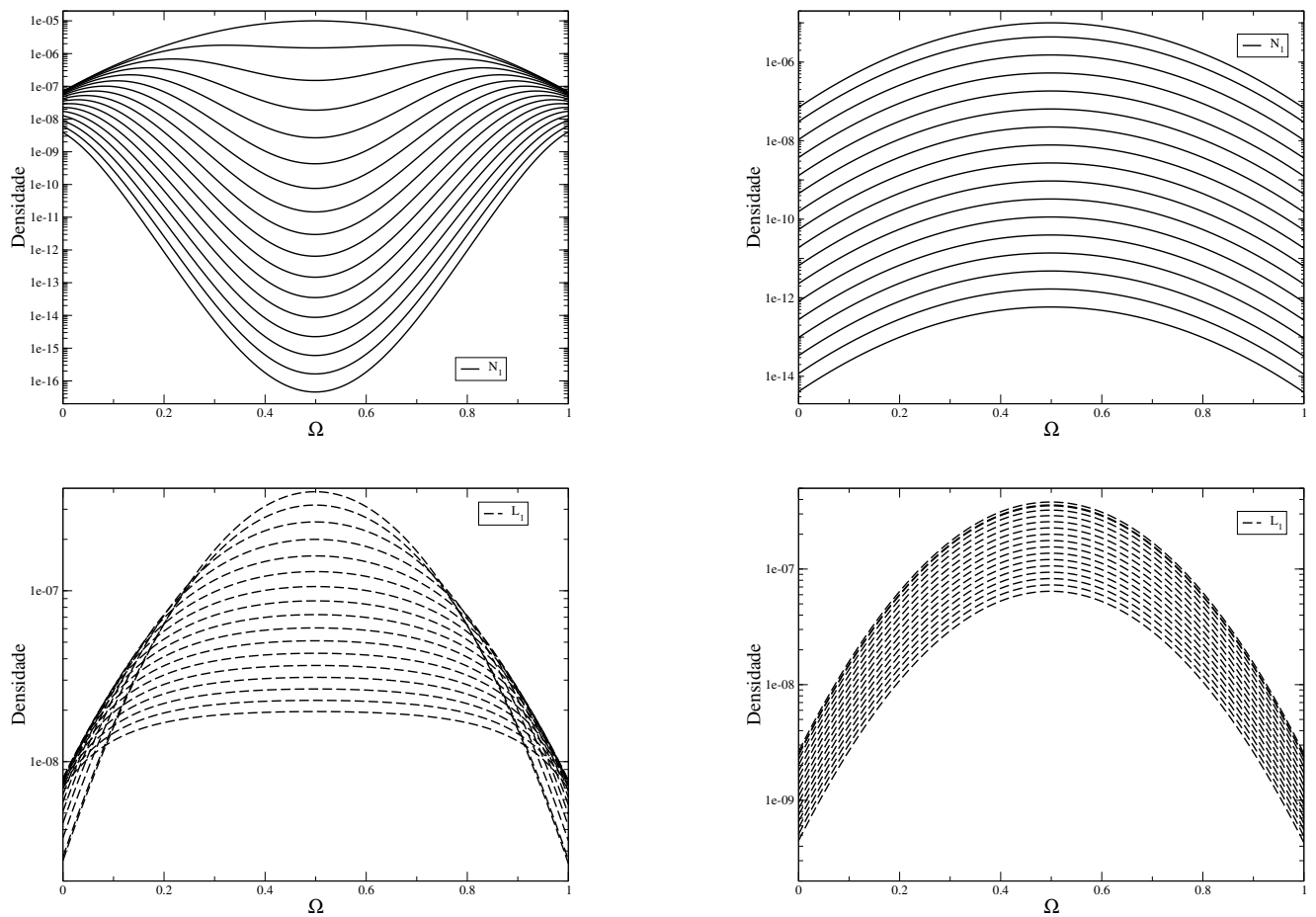

Figura 3: Densidade distribuída espacialmente durante o tratamento segundo o protocolo metronômico, à esquerda para o método convencional e à direta, método uniforme. 


\section{Agradecimentos}

RTG: CAPES; PFAM: Processo 2013/08133-0, Fundação de Amparo à Pesquisa do Estado de São paulo (FAPESP).

\section{Referências}

[1] A. R. A. Anderson and M. A. J. Chaplain, Continuous and discrete mathematical models of tumor-induced angiogenesis, Bull. Math. Biol., vol. 60, 857-899, (1998).

[2] R. Martin and K. L. Teo, Optimal control of drug administration in cancer chemotherapy, World Scientific, Singapore, (1993).

[3] D. S. Rodrigues and P. F. A. Mancera, Mathematical analysis and simulations involving chemotherapy and surgery on large human tumours under a suitable cell-kill functional response, Math. Biosc. Eng., vol. 10, 221-234, (2013). 